Supplement of Biogeosciences, 14, 415-430, 2017

http://www.biogeosciences.net/14/415/2017/

doi:10.5194/bg-14-415-2017-supplement

(C) Author(s) 2017. CC Attribution 3.0 License.

(c) (i)

Supplement of

\title{
Decoupled carbonate chemistry controls on the incorporation of boron into Orbulina universa
}

Ella L. Howes et al.

Correspondence to: Ella L. Howes (ella.1.howes@gmail.com)

The copyright of individual parts of the supplement might differ from the CC-BY 3.0 licence. 
Supplementary Table 1: Individual Orbulina universa $\delta^{11} \mathrm{~B}$ displayed with boron isotopic composition of the seawater medium and seawater chemistry.

\begin{tabular}{|c|c|c|c|c|}
\hline $\begin{array}{c}\mathrm{CO}_{3}{ }^{2-} \\
\left(\mu \mathrm{mol} \mathrm{kg}{ }^{-1}\right)\end{array}$ & $\mathbf{p H}_{\mathrm{T}}$ & $\begin{array}{c}\mathrm{HCO}_{3}^{-} \\
\left(\mu \mathrm{mol} \mathrm{kg}{ }^{-1}\right)\end{array}$ & $\begin{array}{c}\text { Isotopic } \\
\text { composition } \mathrm{H}_{2} \mathrm{O}\end{array}$ & $\begin{array}{l}\delta^{11} B \\
(\% o)\end{array}$ \\
\hline 285 & 8.05 & 2371 & 37.63 & 21.16 \\
\hline 285 & 8.05 & 2371 & 37.63 & 20.97 \\
\hline 285 & 8.05 & 2371 & 37.63 & 21.00 \\
\hline 285 & 8.05 & 2371 & 37.63 & 21.18 \\
\hline 285 & 8.05 & 2371 & 37.63 & 17.94 \\
\hline 285 & 8.05 & 2371 & 4.98 & 18.32 \\
\hline 285 & 8.05 & 2371 & 4.98 & 19.03 \\
\hline 285 & 8.05 & 2371 & 4.98 & 20.30 \\
\hline 285 & 8.05 & 2371 & 4.98 & 20.57 \\
\hline 285 & 8.05 & 2371 & 4.98 & 19.10 \\
\hline 285 & 8.05 & 2371 & 4.98 & 19.62 \\
\hline 285 & 8.05 & 2371 & 4.98 & 19.10 \\
\hline 285 & 8.05 & 2371 & 4.98 & 19.62 \\
\hline 238 & 8.05 & 1981 & 4.98 & 19.22 \\
\hline 238 & 8.05 & 1981 & 4.98 & 19.98 \\
\hline 238 & 8.05 & 1981 & 4.98 & 17.54 \\
\hline 238 & 8.05 & 1981 & 4.98 & 18.07 \\
\hline 238 & 8.05 & 1981 & 4.98 & 17.50 \\
\hline 238 & 8.05 & 1981 & 5.35 & 17.81 \\
\hline 238 & 8.05 & 1981 & 5.35 & 18.18 \\
\hline 238 & 8.05 & 1981 & 5.35 & 17.79 \\
\hline 238 & 8.05 & 1981 & 5.35 & 21.31 \\
\hline 610 & 8.05 & 5069 & 5.35 & 19.49 \\
\hline 610 & 8.05 & 5069 & 5.35 & 19.02 \\
\hline 610 & 8.05 & 5069 & 5.35 & 19.88 \\
\hline 610 & 8.05 & 5069 & 5.35 & 18.64 \\
\hline 610 & 8.05 & 5069 & 5.35 & 23.10 \\
\hline 610 & 8.05 & 5069 & 4.2 & 19.96 \\
\hline 610 & 8.05 & 5069 & 4.2 & 22.57 \\
\hline 610 & 8.05 & 5069 & 4.2 & 22.96 \\
\hline 610 & 8.05 & 5069 & 4.2 & 21.33 \\
\hline 610 & 8.05 & 5069 & 4.2 & 22.32 \\
\hline 610 & 8.05 & 5069 & 4.2 & 22.42 \\
\hline 610 & 8.05 & 5069 & 4.2 & 17.75 \\
\hline 296 & 7.9 & 3478 & 4.2 & 17.19 \\
\hline 296 & 7.9 & 3478 & 4.2 & 16.55 \\
\hline 296 & 7.9 & 3478 & 4.2 & 16.58 \\
\hline 296 & 7.9 & 3478 & 4.2 & 15.82 \\
\hline 296 & 7.9 & 3478 & 4.2 & 17.42 \\
\hline
\end{tabular}




\begin{tabular}{|c|c|c|c|c|}
\hline 296 & 7.9 & 3478 & 4.11 & 15.98 \\
\hline 296 & 7.9 & 3478 & 4.11 & 16.80 \\
\hline 296 & 7.9 & 3478 & 4.11 & 15.80 \\
\hline 296 & 7.9 & 3478 & 4.11 & 18.02 \\
\hline 296 & 7.9 & 3478 & 4.11 & 18.56 \\
\hline 296 & 7.9 & 3478 & 4.11 & 18.66 \\
\hline 296 & 7.9 & 3478 & 4.11 & 18.58 \\
\hline 296 & 7.9 & 3478 & 4.11 & 20.35 \\
\hline 296 & 7.9 & 3478 & 4.11 & 16.19 \\
\hline 296 & 7.9 & 3478 & 4.11 & 18.80 \\
\hline 296 & 7.9 & 3478 & 4.11 & 18.67 \\
\hline 296 & 7.9 & 3478 & 4.11 & 18.79 \\
\hline 296 & 7.9 & 3478 & 4.11 & 18.55 \\
\hline 258 & 7.7 & 4791 & 4.11 & 15.86 \\
\hline 258 & 7.7 & 4791 & 4.11 & 15.74 \\
\hline 258 & 7.7 & 4791 & 4.11 & 16.71 \\
\hline 258 & 7.7 & 4791 & 4.11 & 15.38 \\
\hline 258 & 7.7 & 4791 & 4.11 & 16.04 \\
\hline 258 & 7.7 & 4791 & 4.69 & 14.72 \\
\hline 258 & 7.7 & 4791 & 4.69 & 13.59 \\
\hline 258 & 7.7 & 4791 & 4.69 & 11.86 \\
\hline 258 & 7.7 & 4791 & 4.69 & 14.80 \\
\hline 258 & 7.7 & 4791 & 4.69 & 15.99 \\
\hline 258 & 7.7 & 4791 & 4.69 & 17.04 \\
\hline 258 & 7.7 & 4791 & 4.69 & 16.91 \\
\hline 258 & 7.7 & 4791 & 4.69 & 17.91 \\
\hline 258 & 7.7 & 4791 & 4.69 & 16.78 \\
\hline 258 & 7.7 & 4791 & 4.69 & 15.76 \\
\hline 258 & 7.7 & 4791 & 4.69 & 17.91 \\
\hline 258 & 7.7 & 4791 & 4.69 & 16.78 \\
\hline 258 & 7.7 & 4791 & 4.69 & 15.76 \\
\hline
\end{tabular}


Supplementary Table 2: Individual Orbulina universa B/Ca values displayed with seawater chemistry.

\begin{tabular}{|c|c|c|c|}
\hline $\begin{array}{c}\mathrm{CO}_{3}{ }^{2-} \\
\left(\mu \mathrm{mol} \mathrm{kg}{ }^{-1}\right)\end{array}$ & $\mathbf{p H}_{\mathrm{T}}$ & $\begin{array}{c}\mathrm{HCO}_{3}^{-} \\
\left(\mu \mathrm{mol} \mathrm{kg}{ }^{-1}\right)\end{array}$ & $\mathrm{B} / \mathrm{Ca}$ \\
\hline 239 & 8.05 & 1984 & 1.63 \\
\hline 239 & 8.05 & 1984 & 1.66 \\
\hline 239 & 8.05 & 1984 & 1.45 \\
\hline 239 & 8.05 & 1984 & 1.43 \\
\hline 239 & 8.05 & 1984 & 1.63 \\
\hline 239 & 8.05 & 1984 & 1.14 \\
\hline 239 & 8.05 & 1984 & 1.89 \\
\hline 239 & 8.05 & 1984 & 1.77 \\
\hline 239 & 8.05 & 1984 & 1.54 \\
\hline 239 & 8.05 & 1984 & 0.16 \\
\hline 239 & 8.05 & 1984 & 1.60 \\
\hline 239 & 8.05 & 1984 & 1.73 \\
\hline 239 & 8.05 & 1984 & 1.14 \\
\hline 239 & 8.05 & 1984 & 1.30 \\
\hline 239 & 8.05 & 1984 & 2.19 \\
\hline 239 & 8.05 & 1984 & 1.25 \\
\hline 239 & 8.05 & 1984 & 1.54 \\
\hline 239 & 8.05 & 1984 & 2.16 \\
\hline 239 & 8.05 & 1984 & 1.75 \\
\hline 239 & 8.05 & 1984 & 1.42 \\
\hline 239 & 8.05 & 1984 & 1.57 \\
\hline 239 & 8.05 & 1984 & 1.21 \\
\hline 239 & 8.05 & 1984 & 1.56 \\
\hline 239 & 8.05 & 1984 & 1.19 \\
\hline 239 & 8.05 & 1984 & 1.31 \\
\hline 239 & 8.05 & 1984 & 1.45 \\
\hline 285 & 8.05 & 2374 & 1.00 \\
\hline 285 & 8.05 & 2374 & 1.07 \\
\hline 285 & 8.05 & 2374 & 1.10 \\
\hline 285 & 8.05 & 2374 & 1.10 \\
\hline 285 & 8.05 & 2374 & 1.21 \\
\hline 285 & 8.05 & 2374 & 1.25 \\
\hline 285 & 8.05 & 2374 & 1.30 \\
\hline 285 & 8.05 & 2374 & 1.32 \\
\hline 285 & 8.05 & 2374 & 1.36 \\
\hline 285 & 8.05 & 2374 & 1.36 \\
\hline 285 & 8.05 & 2374 & 1.52 \\
\hline 285 & 8.05 & 2374 & 1.56 \\
\hline 285 & 8.05 & 2374 & 1.73 \\
\hline
\end{tabular}




\begin{tabular}{|c|c|c|c|}
\hline 285 & 8.05 & 2374 & 1.09 \\
\hline 285 & 8.05 & 2374 & 1.22 \\
\hline 285 & 8.05 & 2374 & 0.82 \\
\hline 285 & 8.05 & 2374 & 1.07 \\
\hline 237 & 8.05 & 1968 & 0.13 \\
\hline 237 & 8.05 & 1968 & 0.12 \\
\hline 237 & 8.05 & 1968 & 0.10 \\
\hline 237 & 8.05 & 1968 & 0.10 \\
\hline 237 & 8.05 & 1968 & 0.11 \\
\hline 534 & 8.05 & 4424 & 0.84 \\
\hline 534 & 8.05 & 4424 & 0.90 \\
\hline 534 & 8.05 & 4424 & 0.84 \\
\hline 534 & 8.05 & 4424 & 0.98 \\
\hline 534 & 8.05 & 4424 & 0.99 \\
\hline 534 & 8.05 & 4424 & 1.10 \\
\hline 534 & 8.05 & 4424 & 0.90 \\
\hline 534 & 8.05 & 4424 & 0.86 \\
\hline 534 & 8.05 & 4424 & 0.74 \\
\hline 534 & 8.05 & 4424 & 0.92 \\
\hline 534 & 8.05 & 4424 & 0.97 \\
\hline 534 & 8.05 & 4424 & 0.46 \\
\hline 534 & 8.05 & 4424 & 0.45 \\
\hline 534 & 8.05 & 4424 & 0.93 \\
\hline 534 & 8.05 & 4424 & 0.66 \\
\hline 534 & 8.05 & 4424 & 0.78 \\
\hline 534 & 8.05 & 4424 & 0.55 \\
\hline 534 & 8.05 & 4424 & 0.62 \\
\hline 534 & 8.05 & 4424 & 0.60 \\
\hline 534 & 8.05 & 4424 & 0.60 \\
\hline 534 & 8.05 & 4424 & 0.82 \\
\hline 534 & 8.05 & 4424 & 0.64 \\
\hline 534 & 8.05 & 4424 & 0.64 \\
\hline 534 & 8.05 & 4424 & 0.71 \\
\hline 534 & 8.05 & 4424 & 0.65 \\
\hline 534 & 8.05 & 4424 & 0.79 \\
\hline 296 & 7.9 & 3481 & 0.91 \\
\hline 296 & 7.9 & 3481 & 0.77 \\
\hline 296 & 7.9 & 3481 & 0.86 \\
\hline 296 & 7.9 & 3481 & 0.97 \\
\hline 296 & 7.9 & 3481 & 1.20 \\
\hline 296 & 7.9 & 3481 & 1.34 \\
\hline 296 & 7.9 & 3481 & 0.87 \\
\hline 296 & 7.9 & 3481 & 0.85 \\
\hline 296 & 7.9 & 3481 & 1.03 \\
\hline 296 & 7.9 & 3481 & 0.76 \\
\hline
\end{tabular}




\begin{tabular}{|l|l|l|l|}
\hline 296 & 7.9 & 3481 & 0.67 \\
\hline 296 & 7.9 & 3481 & 0.92 \\
\hline 296 & 7.9 & 3481 & 0.80 \\
\hline 296 & 7.9 & 3481 & 0.85 \\
\hline 258 & 7.7 & 4793 & 0.70 \\
\hline 258 & 7.7 & 4793 & 0.53 \\
\hline 258 & 7.7 & 4793 & 0.80 \\
\hline 258 & 7.7 & 4793 & 0.72 \\
\hline 258 & 7.7 & 4793 & 0.86 \\
\hline 258 & 7.7 & 4793 & 0.72 \\
\hline 258 & 7.7 & 4793 & 0.64 \\
\hline 258 & 7.7 & 4793 & 0.62 \\
\hline 258 & 7.7 & 4793 & 1.10 \\
\hline 258 & 7.7 & 4793 & 0.67 \\
\hline 258 & 7.7 & 4793 & 0.57 \\
\hline 258 & 7.7 & 4793 & 0.52 \\
\hline 258 & 7.7 & 4793 & 0.62 \\
\hline 258 & 7.7 & 4793 & 0.53 \\
\hline
\end{tabular}

Supplementary Table 3: $p$-Values of the ANOVA using a Bonferroni test to verify significance of the mean $\delta^{11} \mathrm{~B}$ difference between the three $\left[\mathrm{CO}_{3}{ }^{2-}\right]$ treatments. Only the treatments 239 and $534 \mu \mathrm{mol} / \mathrm{kg}$ are significantly different (in bold). The overall ANOVA p-value is 0.00203 .

\begin{tabular}{|c|c|c|c|}
\hline$\left[\mathrm{CO}_{3}{ }^{2-}\right]$ & 239 & 286 & 534 \\
\hline 239 & - & 0.05599 & $\mathbf{0 . 0 0 1 4 4}$ \\
\hline 286 & 0.05599 & - & 0.31894 \\
\hline 534 & $\mathbf{0 . 0 0 1 4 4}$ & 0.31894 & - \\
\hline
\end{tabular}


Supplementary Table 4: Error propagation of the uncertainty in the final $\delta^{11} \mathrm{~B}$ of $O$. universa stemming from the LA analysis of the shells and the culture water analysis using: $2 S E_{\text {O.universa }}(\% 0)=\sqrt{\left(2 S E_{\text {calcite }}\right)^{2}+\left(2 S E_{\text {seawater }}\right)^{2}}$

\begin{tabular}{|c|c|c|c|c|}
\hline $\mathrm{pH}$ & $\mathrm{CO}_{3}{ }^{2-}$ & $2 \mathrm{SE} \mathrm{sw}$ & $\begin{array}{c}2 \mathrm{SE} \\
\text { calcite }\end{array}$ & $\begin{array}{c}2 \mathrm{SE} \\
\text { propagated }\end{array}$ \\
\hline 8,05 & 239 & 0,53 & 1 & 1,13 \\
\hline 8,05 & 286 & 0,85 & 0,7 & 1,10 \\
\hline 8,05 & 534 & 1,03 & 1,1 & 1,51 \\
\hline 7,9 & 297 & 0,94 & 0,7 & 1,17 \\
\hline 7,7 & 258 & 2,4 & 0,8 & 2,53 \\
\hline
\end{tabular}

Supplementary Table 5: Individual and averaged measurements of $\delta^{11} \mathrm{~B}$ in the culture waters for each treatment.

\begin{tabular}{|c|c|c|c|c|}
\hline $\mathbf{p H}$ & {$\left[\mathbf{C O}_{\mathbf{3}}{ }^{2-}\right]$} & $\begin{array}{c}\mathbf{\delta}^{\mathbf{1 1}} \mathbf{B} \\
\text { water }\end{array}$ & $\begin{array}{c}\text { Average } \\
\mathbf{\delta}^{\mathbf{1 1}} \mathbf{B} \text { of } \\
\text { treatment } \\
\text { water }\end{array}$ & $\begin{array}{c}\text { Standard } \\
\text { deviation }\end{array}$ \\
\hline 8.05 & 285.6 & 4.23 & 4.98 & 0.85 \\
\hline 8.05 & 285.6 & 5.98 & & \\
\hline 8.05 & 285.6 & 4.31 & & \\
\hline 8.05 & 285.6 & 5.40 & & \\
\hline 8.05 & 238.7 & 5.77 & 5.35 & 0.53 \\
\hline 8.05 & 238.7 & 4.75 & & \\
\hline 8.05 & 238.7 & 5.52 & & \\
\hline 8.05 & 533.9 & 5.97 & 4.20 & 1.03 \\
\hline 8.05 & 533.9 & 3.69 & & \\
\hline 8.05 & 533.9 & 3.42 & & \\
\hline 8.05 & 533.9 & 4.24 & & \\
\hline 8.05 & 533.9 & 3.68 & & \\
\hline 7.9 & 296.6 & 4.11 & 4.11 & 0.95 \\
\hline 7.9 & 296.6 & 3.00 & & \\
\hline 7.9 & 296.6 & 4.02 & & \\
\hline 7.9 & 296.6 & 5.31 & & \\
\hline 7.7 & 257.8 & 4.78 & 4.69 & 2.41 \\
\hline 7.7 & 257.8 & 5.30 & & \\
\hline 7.7 & 257.8 & 4.00 & & \\
\hline & & & & \\
\hline
\end{tabular}


Supplementary Fig. 1: Histograms of single-foram $\delta^{11} \mathrm{~B}$ measurements for each $\mathrm{pH}$ treatment. The $p$ values from Shapiro-Wilk-tests are all higher than 0.05 and hence approve a normal distribution for all treatments. The box plots also suggest a normal distribution of laser ablation analyses, with averages (black squares) very close to the medians (bars within boxes).
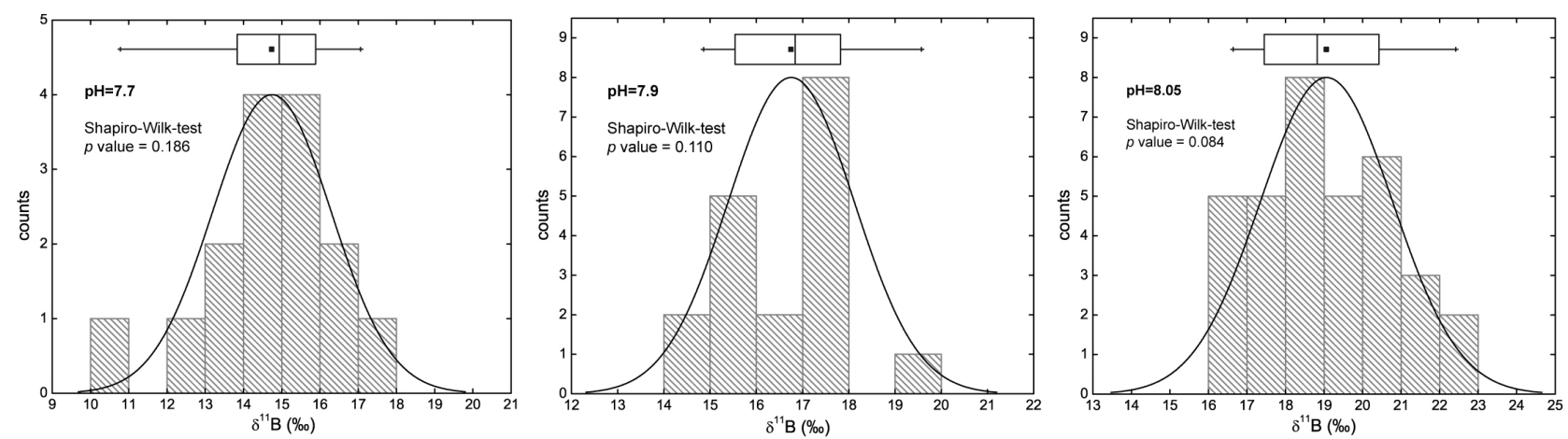
Supplementary Fig. 2: (A) B/Ca $(\mu \mathrm{mol} / \mathrm{mol})$ vs. $\delta^{11} \mathrm{~B}$ per treatment. Each data point corresponds to paired data from one specimen. (B) Mean values for each of the treatments. $\left[\mathrm{CO}_{3}{ }^{2-}\right]$ in $\mu \mathrm{mol}$.
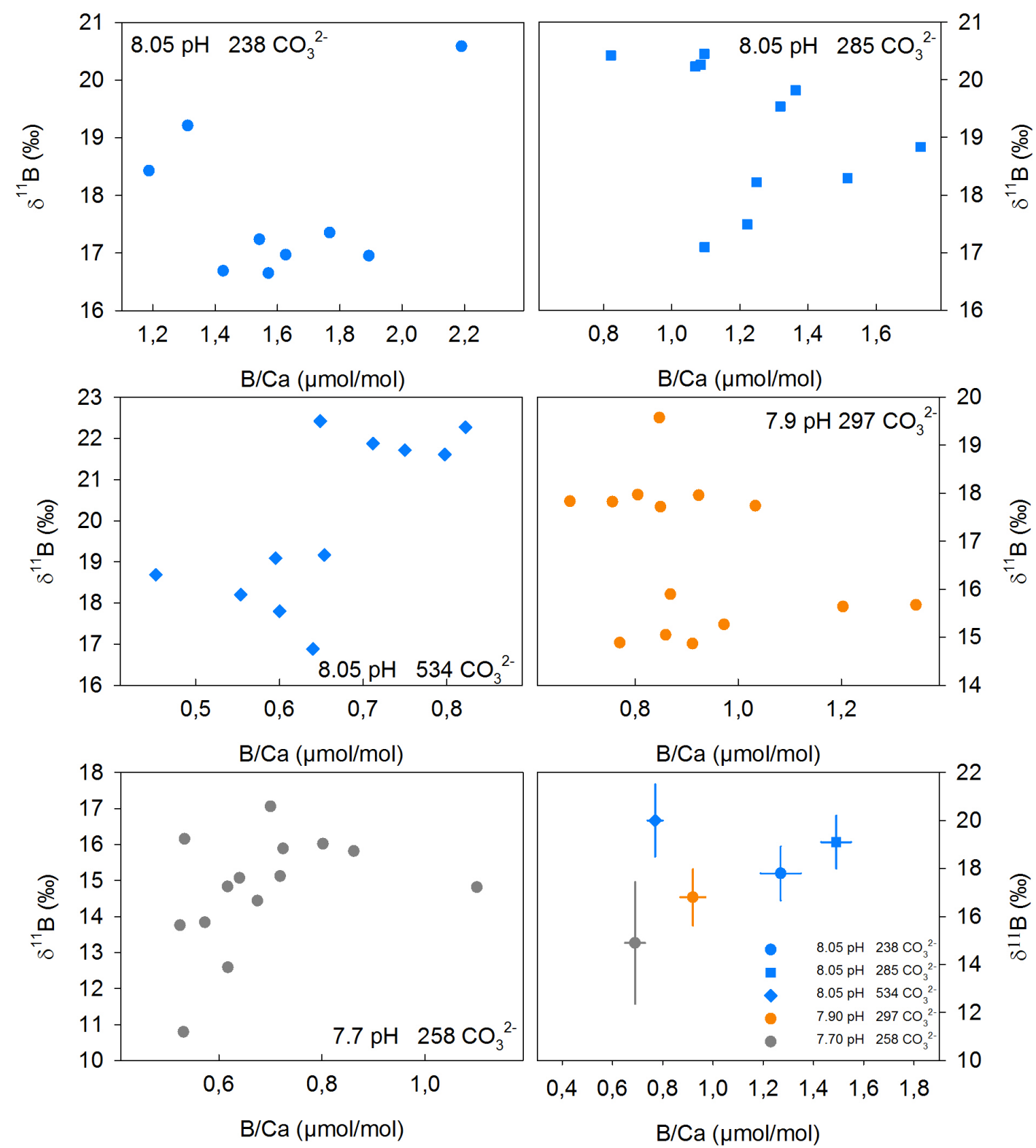